\title{
Permeability Models for Magma Flow through the Earth's Mantle: A Lie Group Analysis
}

\author{
N. Mindu and D. P. Mason \\ Centre for Differential Equations, Continuum Mechanics and Applications and School of \\ Computational and Applied Mathematics, University of the Witwatersrand, Johannesburg, \\ Private Bag 3, Wits 2050, South Africa \\ Correspondence should be addressed to N. Mindu; nkululekomindu@gmail.com
}

Received 29 December 2012; Accepted 1 March 2013

Academic Editor: Fazal M. Mahomed

Copyright (C) 2013 N. Mindu and D. P. Mason. This is an open access article distributed under the Creative Commons Attribution License, which permits unrestricted use, distribution, and reproduction in any medium, provided the original work is properly cited.

The migration of melt through the mantle of the Earth is governed by a third-order nonlinear partial differential equation for the voidage or volume fraction of melt. The partial differential equation depends on the permeability of the medium which is assumed to be a function of the voidage. It is shown that the partial differential equation admits, as well as translations in time and space, other Lie point symmetries provided the permeability is either a power law or an exponential law of the voidage or is a constant. A rarefactive solitary wave solution of the partial differential equation is derived in the form of a quadrature for the exponential law for the permeability.

\section{Introduction}

The one-dimensional migration of melt upwards through the mantle of the Earth is governed by the third-order nonlinear partial differential equation

$$
\frac{\partial \phi}{\partial t}+\frac{\partial}{\partial z}\left[K(\phi)\left(1-\frac{\partial^{2} \phi}{\partial t \partial z}\right)\right]=0
$$

where $\phi(t, z)$ is the voidage or volume fraction of melt, $t$ is time, $z$ is the vertical spatial coordinate, and $K$ is the permeability of the medium. The special case in which $K(\phi)$ is a power law,

$$
K(\phi)=\phi^{n},
$$

has been studied extensively, and solitary wave solutions have been derived [1-12]. In this paper $K(\phi)$ will initially not be specified. For arbitrary forms of $K(\phi)$, (1) does not depend explicitly on $t$ and $z$, and therefore it admits the Lie point symmetries

$$
X_{1}=\frac{\partial}{\partial t}, \quad X_{2}=\frac{\partial}{\partial z} \text {. }
$$

We will determine the forms of $K(\phi)$ for (1) to admit other Lie point symmetries besides the Lie point symmetries (3). This would be a significant property for (1) to posses because invariant solutions could then be constructed. One of the forms obtained for $K(\phi)$ is an exponential law relating the permeability to the voidage. We will derive a new rarefactive solitary wave solution of (1) with the exponential law for the permeability.

The variables $\phi, t, z$, and $K(\phi)$ in (1) are dimensionless. The voidage $\phi(t, z)$ is scaled by the background voidage $\phi_{0}$. The background state is therefore defined by $\phi=1$. The characteristic length in the $z$-direction, which is vertically upwards, is the compaction length $\delta_{c}$ defined by

$$
\delta_{c}=\left[\frac{K\left(\phi_{0}\right)(\xi+(4 / 3) \eta)}{\mu}\right]^{1 / 2}
$$

where $\mu$ is the coefficient of shear viscosity of the melt and $\xi$ and $\eta$ are the bulk and shear viscosity of the solid matrix, respectively. We will assume that $\xi$ and $\eta$ are constants as did Barcilon and Richter [3]. Scott and Stevenson [1] assume that 
$\xi$ and $\eta$ are power laws of the voidage $\phi$. The characteristic time is $t_{0}$ defined by

$$
t_{0}=\frac{\phi_{0}}{g \Delta \rho}\left[\frac{\mu(\xi+(4 / 3)) \eta}{K\left(\phi_{0}\right)}\right]^{1 / 2},
$$

where $g$ is the acceleration due to gravity and $\Delta \rho$ is the difference between the density of the solid matrix and the density of the melt. The permeability is scaled by $K\left(\phi_{0}\right)$ and therefore

$$
K(1)=1
$$

When the voidage is zero, the permeability must also be zero and therefore

$$
K(0)=0 .
$$

In the derivation of (1), it is assumed that the background voidage satisfies $\phi_{0} \ll 1$. An outline of the derivation of (1) when $K(\phi)$ satisfies the power law (2) is given by Nakayama and Mason [5]. The derivation is readily extended to the general case in which $K=K(\phi)$.

An outline of the paper is as follows. In Section 2 the Lie point symmetries of (1) are investigated and the forms of $K(\phi)$ are determined for (1) to admit, as well as the Lie point symmetries (3), other Lie point symmetries. In Section 3 a new rarefactive solitary wave solution of (1) is obtained when $K(\phi)$ depends on $\phi$ through an exponential law. Finally, the conclusions are summarized in Section 4.

\section{Lie Point Symmetries}

In this section $K(\phi)$ will not be specified initially. We will investigate the Lie point symmetries of (1) and the forms of $K(\phi)$ for these symmetries to exist.

Equation (1) is as follows:

$$
\phi_{t}+\frac{d K}{d \phi} \phi_{z}-\frac{d K}{d \phi} \phi_{z} \phi_{t z}-K \phi_{t z z}=0,
$$

where a subscript denotes partial differentiation. We look for Lie point symmetries of the form

$$
X=\xi^{1}(t, z, \phi) \frac{\partial}{\partial t}+\xi^{2}(t, z, \phi) \frac{\partial}{\partial z}+\eta(t, z, \phi) \frac{\partial}{\partial \phi} .
$$

The coefficients $\xi$ and $\eta$ in the Lie point symmetry (9) should not be confused with the bulk and shear viscosities in the characteristic quantities (4) and (5). The invariance criterion is

$$
\left.X^{[3]}\left(\phi_{t}+\frac{d K}{d \phi} \phi_{z}-\frac{d K}{d \phi} \phi_{z} \phi_{t z}-K \phi_{t z z}\right)\right|_{(8)}=0,
$$

where the third prolongation of $X$ is of the form

$$
\begin{aligned}
X^{[3]}= & \xi^{1} \frac{\partial}{\partial t}+\xi^{2} \frac{\partial}{\partial z}+\eta \frac{\partial}{\partial \phi}+\zeta_{1} \frac{\partial}{\partial \phi_{t}}+\zeta_{2} \frac{\partial}{\partial \phi_{z}} \\
& +\zeta_{12} \frac{\partial}{\partial \phi_{t z}}+\zeta_{122} \frac{\partial}{\partial \phi_{t z z}}+\cdots
\end{aligned}
$$

The remaining terms in $X^{[3]}$ are not required in (10) and

$$
\begin{gathered}
\zeta_{i}=D_{i}(\eta)-\phi_{s} D_{i}\left(\xi^{s}\right), \quad i=1,2, \\
\zeta_{i j}=D_{j}\left(\zeta_{i}\right)-\phi_{i s} D_{j}\left(\xi^{s}\right), \quad i=1,2, \\
\zeta_{i j k}=D_{k}\left(\zeta_{i j}\right)-\phi_{i j s} D_{k}\left(\xi^{s}\right), \quad i=1,2,
\end{gathered}
$$

with summation over the repeated index, $s$, from 1 to 2 . The total derivatives $D_{1}$ and $D_{2}$ are defined by

$$
\begin{aligned}
& D_{1}=D_{t}=\frac{\partial}{\partial t}+\phi_{t} \frac{\partial}{\partial \phi}+\phi_{t t} \frac{\partial}{\partial \phi_{t}}+\phi_{z t} \frac{\partial}{\partial \phi_{z}}+\cdots \\
& D_{1}=D_{z}=\frac{\partial}{\partial z}+\phi_{z} \frac{\partial}{\partial \phi}+\phi_{t z} \frac{\partial}{\partial \phi_{t}}+\phi_{z z} \frac{\partial}{\partial \phi_{z}}+\cdots
\end{aligned}
$$

We replace $\phi_{t z z}$ in the determining equation (10) using the partial differential equation (8) and then separate the determining equation according to the following partial derivatives of $\phi$ :

$$
\begin{gathered}
\phi_{t t z}: \frac{\partial \xi^{1}}{\partial z}=0, \\
\phi_{z} \phi_{t t z}: \frac{\partial \xi^{1}}{\partial \phi}=0, \\
\phi_{z z z}: \frac{\partial \xi^{2}}{\partial t}=0, \\
\phi_{t} \phi_{z z z}: \frac{\partial \xi^{2}}{\partial \phi}=0, \\
\phi_{z z}: \frac{\partial^{2} \eta}{\partial t \partial \phi}=0, \\
\phi_{t} \phi_{z z}: \frac{\partial^{2} \eta}{\partial \phi^{2}}=0 .
\end{gathered}
$$

It follows directly from (14) that

$$
\xi^{1}=f(t), \quad \xi^{2}=g(z), \quad \eta=\phi A(z)+B(t, z),
$$

where $f(t), g(z), A(z)$, and $B(t, z)$ still have to be determined. Using (15), the invariance criterion separates further into the following system of equations:

$$
\begin{aligned}
& \phi_{z} \phi_{t z}: {\left[\phi \frac{d^{2} K}{d \phi^{2}}-\frac{\phi}{K}\left(\frac{d K}{d \phi}\right)^{2}+\frac{d K}{d \phi}\right] A(z) } \\
&+\left[\frac{d^{2} K}{d \phi^{2}}-\frac{1}{K}\left(\frac{d K}{d \phi}\right)^{2}\right] B(t, z)=0,
\end{aligned}
$$




$$
\begin{aligned}
\phi_{t z}: & {\left[\phi \frac{d K}{d \phi}+2 K(\phi)\right] \frac{d A}{d z}+\frac{d K}{d \phi} \frac{\partial B}{\partial z} } \\
& -K(\phi) \frac{d^{2} g}{d z^{2}}=0 \\
\phi_{t} \phi_{z}: & \frac{d K}{d \phi} \frac{d A}{d z}=0, \\
\phi_{z}: & {\left[\phi \frac{d^{2} K}{d \phi^{2}}-\frac{\phi}{K}\left(\frac{d K}{d \phi}\right)^{2}\right] A(z) } \\
& +\left[\frac{d^{2} K}{d \phi^{2}}-\frac{1}{K}\left(\frac{d K}{d \phi}\right)^{2}\right] B(t, z) \\
& -\frac{d K}{d \phi} \frac{\partial^{2} B}{\partial t \partial z}+\frac{d K}{d \phi}\left(\frac{d f}{d t}+\frac{d g}{d z}\right)=0 \\
\phi_{t}: & K(\phi) \frac{d^{2} A}{d z^{2}}+\frac{\phi}{K} \frac{d K}{d \phi} A(z)+\frac{1}{K} \frac{d K}{d \phi} B(t, z) \\
& -2 \frac{d g}{d z}=0,
\end{aligned}
$$

Remainder: $\phi \frac{d K}{d \phi} \frac{d A}{d z}-K(\phi) \frac{\partial^{3} B}{\partial t \partial z^{2}}+\frac{d K}{d \phi} \frac{\partial B}{\partial z}$

$$
+\frac{\partial B}{\partial t}=0
$$

For arbitrary forms of $K(\phi),(16)$ to (21) are satisfied by

$$
A(z)=0, \quad B(t, z)=0, \quad f(t)=c_{1}, \quad g(z)=c_{2},
$$

where $c_{1}$ and $c_{2}$ are constants and (1) admits the Lie point symmetries (3). We will seek possible forms of $K(\phi)$ for which (1) admits, as well as (3), other Lie point symmetries. From (18) we see that there are two general cases depending on whether the permeability, $K$, depends on $\phi$ or is constant.

2.1. Permeability Depends on Voidage. Consider first the case in which $K(\phi)$ depends on the voidage, $\phi$; that is, the permeability is not constant, so that

$$
\frac{d K}{d \phi} \neq 0
$$

Then, from (18), $A(z)=A_{0}$, where $A_{0}$ is a constant. By differentiating (20) with respect to $t$ we find that $B=B(z)$, and from (21) it follows that $B(z)=B_{0}$, where $B_{0}$ is a constant. Hence, from (15),

$$
\eta(\phi)=A_{0} \phi+B_{0}
$$

From (17),

$$
\xi^{2}=g(z)=c_{1} z+c_{2}
$$

where $c_{1}$ and $c_{2}$ are constants. Also from (16) and (19),

$$
\frac{d f}{d t}=A_{0}-c_{1}
$$

and therefore

$$
\xi^{1}=f(t)=\left(A_{0}-c_{1}\right) t+c_{3}
$$

where $c_{3}$ is a constant. Equations (16) to (21) reduce to

$$
\begin{gathered}
\left(A_{0} \phi+B_{0}\right)\left(\frac{d^{2} K}{d \phi^{2}}-\frac{1}{K}\left(\frac{d K}{d \phi}\right)^{2}\right)+A_{0} \frac{d K}{d \phi}=0 \\
\left(A_{0} \phi+B_{0}\right) \frac{d K}{d \phi}-2 c_{1} K=0 .
\end{gathered}
$$

It is readily verified that if $K(\phi)$ satisfies $(29)$, then $K(\phi)$ satisfies (28) identically. Equation (28) therefore does not need to be considered further.

When (23) is satisfied Lie point symmetries of (1) exist provided that $K(\phi)$ satisfies (29) and are given by (24), (25), and (27). This case separates into two subcases depending on whether $A_{0} \neq 0$ or $A_{0}=0$.

2.1.1. The Case $A_{0} \neq 0$. Consider first $A_{0} \neq 0$. The general solution of the ordinary differential equation (29) for $K(\phi)$ is

$$
K(\phi)=K_{0}\left(A_{0} \phi+B_{0}\right)^{2 c_{1} / A_{0}}
$$

where $K_{0}$ is a constant. But since $K(0)=0$, it follows that $B_{0}=0$, and since $K(1)=1$ we obtain

$$
K(\phi)=\phi^{n}, \quad n=\frac{2 c_{1}}{A_{0}} .
$$

Since $K$ is not a constant, $n \neq 0$. Equations (27), (25), and (24) become

$$
\begin{gathered}
\xi^{1}(t)=\frac{c_{1}}{n}(2-n) t+c_{3}, \\
\xi^{2}(z)=c_{1} z+c_{2}, \\
\eta(\phi)=\frac{2 c_{1}}{n} \phi .
\end{gathered}
$$

The three Lie point symmetries of (1) with the power law (30) for $K(\phi)$ are presented in Table 1 . The results agree with those derived by Maluleke and Mason [7, 9] for the generalized magma equation with $m=0$, where $m$ is the exponent in the power law relating the bulk and shear viscosities of the solid matrix to the voidage.

2.1.2. The Case $A_{0}=0, B_{0} \neq 0$. When $A_{0}=0$ but $B_{0} \neq 0$, the general solution of (29) is

$$
K(\phi)=K_{0} \exp (n \phi), \quad n=\frac{2 c_{1}}{B_{0}},
$$

where $K_{0}$ is a constant. Since $K(\phi)$ is not constant, $n \neq 0$, and because the permeability increases as the voidage increases, $n>0$. Also, $K(1)=1$ and therefore

$$
K(\phi)=\exp (n(\phi-1)), \quad n>0 .
$$


TABLE 1: The permeability $K(\phi)$ and the corresponding Lie point symmetries of (1).

\begin{aligned} \hline Permeability & Lie point symmetry \\ \hline$X_{1} & =(2-n) t \frac{\partial}{\partial t}+n z \frac{\partial}{\partial z}+2 \phi \frac{\partial}{\partial \phi} \\ X_{2} & =\frac{\partial}{\partial z} \\ X_{3} & =\frac{\partial}{\partial t} \\ X_{1} & =-n t \frac{\partial}{\partial t}+n z \frac{\partial}{\partial z}+2 \frac{\partial}{\partial \phi} \\ X_{2} & =\frac{\partial}{\partial z} \\ X_{3} & =\frac{\partial}{\partial t} \\ X_{1} & =f(t) \frac{\partial}{\partial t} \\ X_{2} & =\phi \frac{\partial}{\partial \phi} \\ X_{3} & =\frac{\partial}{\partial z} \\ X_{4} & =\cosh (2 z) \frac{\partial}{\partial z}+\phi \sinh (2 z) \frac{\partial}{\partial \phi} \\ X_{5} & =\sinh (2 z) \frac{\partial}{\partial z}+\phi \cosh (2 z) \frac{\partial}{\partial \phi} \\ X_{6} & =B(t, z) \frac{\partial}{\partial \phi}$, where $B(t, z) \operatorname{satisfies} \\ \frac{\partial B}{\partial t} & =\frac{\partial^{3} B}{\partial t \partial z^{2}}=0 \\ K(\phi)=1 \quad & \end{aligned}$

When $\phi=0$, then

$$
K(\phi)=\exp (-n) \neq 0 .
$$

If $n$ is large, $K(0)$ is small. However, the exponential law (34) for $K(\phi)$ does not satisfy the condition $K(0)=0$. It is not a suitable model for physical phenomena with small values of the voidage. For instance, it would not be suitable to describe compressive solitary waves which contain $\phi=$ $0[8,10-12]$. It is suitable for describing rarefactive solitary waves which satisfy $\phi \geq 1$ and this will be considered in Section 3. Equations (27), (25), and (24) become

$$
\begin{gathered}
\xi^{1}(t)=-c_{1} t+c_{3}, \\
\xi^{2}(z)=c_{1} z+c_{2}, \\
\eta(\phi)=\frac{2 c_{1}}{n} .
\end{gathered}
$$

The three Lie point symmetries of (1) with the exponential law (34) for $K(\phi)$ are presented in Table 1.

2.2. Constant Permeability. Finally, consider constant permeability, $K(\phi)=K_{0}$. Since $K(1)=1$, it follows that $K_{0}=1$. The model does not satisfy $K(0)=0$ and it cannot be used to describe physical phenomena in which the permeability depends on voidage.
When $K(\phi)=1,(16)$ to $(21)$ are reduced to

$$
\begin{aligned}
& 2 \frac{d A}{d z}-\frac{d^{2} g}{d z^{2}}=0, \\
& \frac{d^{2} A}{d z^{2}}-2 \frac{d g}{d z}=0, \\
& \frac{\partial B}{\partial t}-\frac{\partial^{3} B}{\partial t \partial z^{2}}=0 .
\end{aligned}
$$

Integrating (37) and (38) once with respect to $z$ gives

$$
\begin{aligned}
& 2 A(z)-\frac{d g}{d z}=c_{1}, \\
& \frac{d A}{d z}-2 g(z)=c_{2},
\end{aligned}
$$

where $c_{1}$ and $c_{2}$ are constants. By eliminating $A(z)$, we obtain

$$
\frac{d^{2} g}{d z^{2}}-4 g=2 c_{2}
$$

and therefore

$$
g(z)=c_{3} \cosh (2 z)+c_{4} \sinh (2 z)-\frac{c_{2}}{2},
$$

where $c_{3}$ and $c_{4}$ are constants. From (40),

$$
A(z)=c_{3} \sinh (2 z)+c_{4} \cosh (2 z)+\frac{c_{1}}{2} .
$$

Thus, from (15)

$$
\begin{gathered}
\xi^{1}(t)=f(t), \\
\xi^{2}(z)=c_{3} \cosh (2 z)+c_{4} \sinh (2 z)-\frac{c_{2}}{2}, \\
\eta(t, z, \phi)=\phi\left(c_{3} \sinh (2 z)+c_{4} \cosh (2 z)+\frac{c_{1}}{2}\right)+B(t, z),
\end{gathered}
$$

where $f(t)$ is arbitrary and $B(t, z)$ satisfies (39) which is the partial differential equation (1) with $K(\phi)=1$. The Lie point symmetries of (1) with constant permeability are presented in Table 1 . The results agree with those derived by Maluleke and Mason [7,9] for $n=0$ and $m=0$.

There are therefore three forms of $K(\phi)$ for which (1) has Lie point symmetries in addition to (3), namely, the power law (31), the exponential law (34), and constant permeability.The Lie point symmetries of (1) with the three forms of $K(\phi)$ are presented in Table 1. Equation (1) with the power law has been studied in detail. In Section 3 we will consider the exponential law and investigate rarefactive solitary wave solutions of (1) with $K(\phi)$ given by (34).

\section{Rarefactive Solitary Wave}

When the permeability satisfies the exponential law (34), the partial differential equation (1) becomes

$$
\frac{\partial \phi}{\partial t}+\frac{\partial}{\partial z}\left[\exp [n(\phi-1)]\left(1-\frac{\partial^{2} \phi}{\partial t \partial z}\right)\right]=0
$$


We now derive a rarefactive solitary wave solution, with $\phi \geq 1$, of the partial differential equation (46).

The solution $\phi=\Phi(t, z)$ is an invariant solution of (46) provided that

$$
\left.X(\phi-\Phi(t, z))\right|_{\phi=\Phi}=0,
$$

where $X$ is a linear combination of the Lie point symmetries of (46). The Lie point symmetries of (46) are given in Table 1. Consider the invariant solution generated by the linear combination

$$
X=c_{1} \frac{\partial}{\partial t}+c_{2} \frac{\partial}{\partial z}
$$

(47) becomes

$$
c_{1} \frac{\partial \Phi}{\partial t}+c_{2} \frac{\partial \Phi}{\partial z}=0
$$

where $c_{1}$ and $c_{2}$ are constants. The general solution of (49) is readily derived, and since $\phi=\Phi(t, z)$ we obtain

$$
\phi=\psi(\zeta), \quad \zeta=z-c t,
$$

where $c=c_{2} / c_{1}$. The group invariant solution (50) is a travelling wave solution and the constant $c$ is the dimensionless speed of the wave.

Substitute (50) into (46). This gives the third-order ordinary differential equation

$$
c \frac{d \psi}{d \zeta}-\frac{d}{d \zeta}\left[\exp [n(\psi-1)]\left(1+c \frac{d^{2} \psi}{d \zeta^{2}}\right)\right]=0,
$$

and integrating (51) once with respect to $\xi$ we obtain

$$
c \frac{d^{2} \psi}{d \zeta^{2}}=\frac{A}{\exp [n(\psi-1)]}+\frac{c \psi}{\exp [n(\psi-1)]}-1,
$$

where $A$ is a constant of integration. Since the right hand side of (52) depends only on $\psi$, we integrate (52) with respect to $\psi$. Now

$$
\frac{d^{2} \psi}{d \zeta^{2}}=\frac{1}{2} \frac{d}{d \psi}\left(\left(\frac{d \psi}{d \zeta}\right)^{2}\right)
$$

and (52) becomes

$$
\frac{c}{2} \frac{d}{d \psi}\left(\left(\frac{d \psi}{d \zeta}\right)^{2}\right)=\frac{A}{\exp [n(\psi-1)]}+\frac{c \psi}{\exp [n(\psi-1)]}-1,
$$

but

$$
\begin{aligned}
& \int^{\psi} \frac{d \psi}{\exp [n(\psi-1)]}=-\frac{1}{n \exp [n(\psi-1)]}, \\
& \int^{\psi} \frac{d \psi}{\exp [n(\psi-1)]}=-\frac{(1+n \psi)}{n^{2} \exp [n(\psi-1)]},
\end{aligned}
$$

and integrating (54) once with respect to $\psi$, we obtain

$$
\begin{aligned}
\frac{c}{2}\left(\frac{d \psi}{d \zeta}\right)^{2}= & -\frac{A}{n \exp [n(\psi-1)]} \\
& -\frac{c(1+n \psi)}{n^{2} \exp [n(\psi-1)]}-\psi+B,
\end{aligned}
$$

where $B$ is a constant.

In order to obtain the three arbitrary constants, $A, B$, and $c$, we impose three boundary conditions suitable for a rarefactive solitary wave. The background state is $\psi=1$. Three boundary conditions for a rarefactive solitary wave are

$$
\begin{aligned}
& \psi=1: \frac{d \psi}{d \zeta}=0, \quad \frac{d^{2} \psi}{d \zeta^{2}}=0, \\
& \psi=\Psi: \frac{d \psi}{d \zeta}=0 .
\end{aligned}
$$

The amplitude of the solitary wave is $\Psi-1$ and $\psi(\zeta)$ has a local maximum when $\psi=\Psi$. Using (52) and (56), the three boundary conditions give

$$
\begin{gathered}
\frac{A}{n}+\frac{(1+n) c}{n^{2}}+1-B=0, \\
A+c-1=0, \\
\frac{A}{n \exp [n(\Psi-1)]}+\frac{c(1+n \Psi)}{n^{2} \exp [n(\Psi-1)]}+\Psi-B=0 .
\end{gathered}
$$

Hence,

$$
c=n\left[\frac{(n(\Psi-1)-1) \exp [n(\Psi-1)]+1}{\exp [n(\Psi-1)]-n(\Psi-1)-1}\right]
$$

and, expressed in terms of $c$,

$$
A=1-c, \quad B=\frac{1}{n^{2}}\left(c+n+n^{2}\right) .
$$

Equation (56) becomes

$$
\left(\frac{d \psi}{d \zeta}\right)^{2}=f(\psi)
$$

where

$$
\begin{aligned}
f(\psi)=\frac{2}{c n^{2}}\left[n+c-n^{2}(\psi-1)\right. \\
-(n+c+n c(\psi-1)) \exp [-n(\psi-1)]] .
\end{aligned}
$$

Now from (53) and the boundary conditions (57),

$$
f(1)=0, \quad \frac{d f}{d \psi}(1)=0, \quad f(\Psi)=0,
$$

and since the left hand side of (61) is nonnegative, for a solitary wave to exist it is necessary that $f(\psi)>0$ for $1<\psi<\Psi$. 
Before proceeding further with the solution, we first investigate the properties of $f(\psi)$. In order to do that we define

$$
F(\psi)=n\left[\frac{(n(\psi-1)-1) \exp [n(\psi-1)]+1}{\exp [n(\psi-1)]-n(\psi-1)-1}\right] .
$$

From (59), it follows that

$$
F(\Psi)=c .
$$

Equation (62) can be written in terms of $F(\psi)$ as follows

$$
\begin{aligned}
f(\psi)= & \frac{2}{n^{2}}[1-(1+n(\psi-1)) \exp [-n(\psi-1)]] \\
& \times\left[\frac{F(\Psi)-F(\psi)}{F(\Psi)}\right]
\end{aligned}
$$

Now,

$$
\begin{aligned}
\frac{d F}{d \psi}= & \frac{4 n^{2} \exp [n(\psi-1)]}{[\exp [n(\psi-1)]-n(\psi-1)-1]^{2}} \\
& \times\left[\sinh ^{2}\left(\frac{n(\psi-1)}{2}\right)-\left(\frac{n(\psi-1)}{2}\right)^{2}\right]
\end{aligned}
$$

and since $\sinh ^{2} x>x^{2}$ for $x \neq 0$, it follows that for $n \neq 0$ and $\psi \neq 1$,

$$
\frac{d F}{d \psi}>0
$$

Hence, $F(\psi)$ is an increasing function of $\psi$. Also,

$$
F(1)=n \text {. }
$$

Consider first $n>0$. Since $F(\psi)$ is an increasing function of $\psi$, it follows that $F(\psi)>n>0$ and $F(\Psi)-F(\psi)>0$ for $1<\psi<\Psi$. Also, it can be verified that

$$
1-\frac{(1+n(\psi-1))}{\exp [n(\psi-1)]}>0,
$$

for $\psi>1$. Hence from (66), $f(\psi)>0$ for $1<\psi<\Psi$ and a rarefactive solitary wave solution exists. Consider next $n<0$. Then from (64), $F(\psi) \rightarrow 0$ as $\psi \rightarrow \infty$, and since $F(1)=$ $n<0$ and $F(\psi)$ is an increasing function of $\psi$, it follows that $F(\Psi)<0$. We still have $F(\Psi)-F(\psi)>0$ by $(68)$ and $(70)$ is still satisfied for $n<0$. Hence from (66), $f(\psi)<0$ for $1<\psi<\Psi$ and a rarefactive solitary wave solution does not exist.

The difference between $F(\psi)$ for $n>0$ and $n<0$ is illustrated in Figure 1 for $n=-0.1,0.5,1$, and 2. For $n<0$ the permeability decreases as the voidage, $\phi$, increases, which is generally not observed physically. When the permeability satisfies the power law (30), a rarefactive solitary wave solution exists for $n>1$ and does not exist for $0 \leq n \leq 1$ [5].

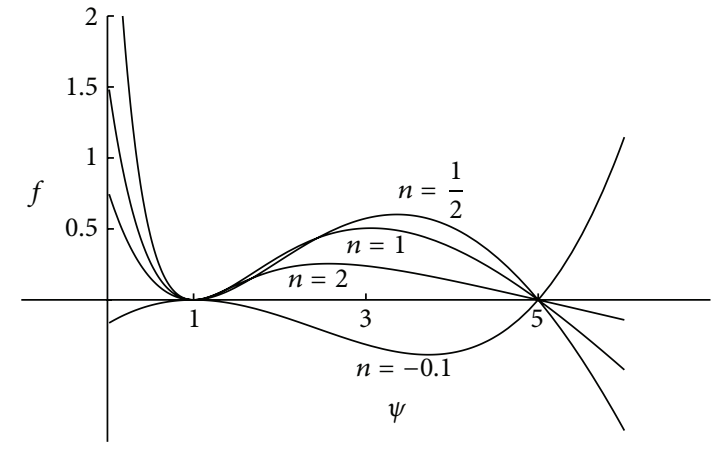

Figure 1: The function $f(\psi)$ defined by (62) plotted against $\psi$ for $n=-0.1,0.5,1$, and 2 for $\Psi=5$.

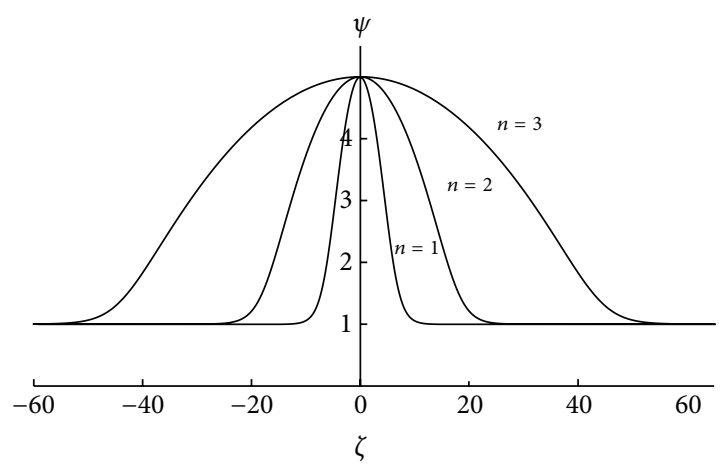

FIGURE 2: Rarefactive solitary wave for $n=1,2$, and 3 with $\Psi=5$. The length $\zeta$ is scaled with the characteristic length $\delta_{c}$ for $n=1$.

Now from (61) and (62), the rarefactive solitary wave solution is given by the following:

$$
\begin{aligned}
\zeta= \pm n\left(\frac{c}{2}\right)^{2} & \\
\times \int_{\psi}^{\Psi}\left((d \psi) \times\left(\left[n+c-n^{2}(\psi-1)\right.\right.\right. & -(n+c+n c(\psi-1)) \\
& \left.\left.\times \exp (-n(\psi-1))]^{1 / 2}\right)^{-1}\right),
\end{aligned}
$$

where the wave speed $c$ is given by (59). In Figure 2, the solitary wave solution (71) is plotted against $\zeta$ for a range of values of $n$. In order to compare the solutions for different values of $n$, the length $\zeta$ is scaled in all cases with the same characteristic length, namely, $\delta_{c}$ calculated for $n=1$. We see that the width of the solitary wave increases as $n$ increases. The increase in the permeability has the effect of spreading the solitary wave. 


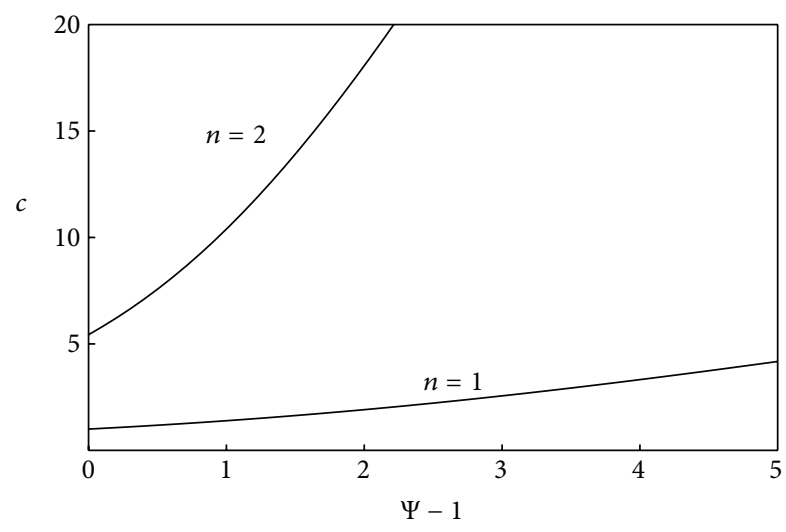

FIGURE 3: Velocity, $c$, of a rarefactive solitary wave for $n=1$ and 2 plotted against amplitude $\Psi-1$. Both velocities are scaled by the characteristic velocity, $\delta_{c} / t_{0}$, for $n=1$.

Consider now the dependence of the wave velocity, $c$, on the amplitude of the solitary wave, $\Psi-1$. Since $F(\Psi)=c$, it follows from (68) that

$$
\frac{d c}{d \Psi}>0
$$

The velocity increases as the amplitude of the solitary wave increases, and therefore larger amplitude waves travel faster. This property also holds for the solitary waves described by the power law (31) for $n>1$ [5]. Equation (72) is a special case of the general result that $F(\psi)$ is an increasing function of $\psi$ for $1 \leq \psi \leq \Psi$ which was central to the proof of the existence of solitary wave solutions for $n>0$. There is therefore a close connection between the property that larger amplitude solitary waves travel faster and the existence of solitary wave solutions. We also determine from (69) the limiting value

$$
\Psi-1=0: c=n .
$$

This limiting value also holds for the dimensionless velocity of rarefactive solitary waves for the power law (31) with $n>1$.

In Figure 3, the dimensionless wave velocity, $c$, of the solitary wave, given by (59), is plotted against the amplitude $\Psi-1$ for $n=1$ and 2. In order to compare $c$ for different values of $n$, the wave velocity is scaled for each value of $n$ by the same characteristic velocity $v_{0}=\delta_{c} / t_{0}$ calculated for $n=1$. We see that $c$ increases steadily with $\Psi-1$ for both cases in agreement with (72). We also see that as $n$ increases the velocity $c$ increases. The increase in the permeability as $n$ increases allows the melt to propagate at a greater speed through the solid matrix.

\section{Conclusions}

One of the functional forms which the permeability $K(\phi)$ must satisfy for the partial differential equation (1) to possess Lie point symmetries besides translation in time and space is the power law. This relation between the permeability and the voidage has been studied extensively to model the migration of melt through the mantle of the Earth. Mainly, travelling wave solutions have been considered, but more general group invariant solutions which include the third Lie point symmetry have been investigated by Harris and Clarkson [8].

Unlike the power law, the exponential law relating the permeability to the voidage is not valid at $\phi=0$ because, when the voidage vanishes, the permeability should also vanish. It can be used when the migration of melt through the Earth's mantle is modelled as a rarefactive solitary wave with $\phi \geq 1$. For both the power law and exponential law, larger amplitude waves travel faster. There is a close connection between this property and the existence of solitary wave solutions.

The conservation laws for the partial differential equation (1) with the power law for $K(\phi)$ have been investigated by several authors $[3,6,9]$. The number of conservation laws is finite, except possibly for $n=-1$ which is not physical. This indicates that the solitary waves are not solitons. The conservation laws for the partial differential equation (46) with the exponential law for $K(\phi)$ still need to be investigated. If it is found that the number of conservation laws is finite it would indicate that the rarefactive solitary waves with the exponential law are also not solitons.

We have assumed that the bulk viscosity, $\xi$, and shear viscosity, $\eta$, of the solid matrix are constant. A large amount of research has been performed on models in which $\xi+(4 / 3) \eta$ is related to the voidage by a power law with exponent $m[1,4-$ 12]. The Lie group analysis for the permeability considered here could be extended to include the viscosity of the solid matrix.

\section{Acknowledgments}

N. Mindu thanks Shanduka and Tomorrow Trust for financial support. D. P. Mason thanks the National Research Foundation, Pretoria, South Africa, for financial support. The authors thank a referee for going through the paper thoroughly and suggesting improvements to the text.

\section{References}

[1] D. R. Scott and D. J. Stevenson, "Magma solitons," Geophysical Research Letters, vol. 4, pp. 1161-1164, 1984.

[2] D. Mckenzie, "The generation and compaction of partially molten rock," Journal of Petrology, vol. 25, no. 3, pp. 713-765, 1984.

[3] V. Barcilon and F. M. Richter, "Nonlinear waves in compacting media," Journal of Fluid Mechanics, vol. 164, pp. 429-448, 1986.

[4] D. Takahashi and J. Satsuma, "Explicit solutions of magma equation," Journal of the Physical Society of Japan, vol. 57, no. 2, pp. 417-421, 1988.

[5] M. Nakayama and D. P. Mason, "Rarefactive solitary waves in two-phase fluid flow of compacting media," Wave Motion, vol. 15, no. 4, pp. 357-392, 1992.

[6] S. E. Harris, "Conservation laws for a nonlinear wave equation," Nonlinearity, vol. 9, no. 1, pp. 187-208, 1996.

[7] G. H. Maluleke and D. P. Mason, "Optimal system and group invariant solutions for a nonlinear wave equation," Communications in Nonlinear Science and Numerical Simulation, vol. 9, no. 1, pp. 93-104, 2004. 
[8] S. E. Harris and P. A. Clarkson, "Painlevé analysis and similarity reductions for the magma equation," Symmetry, Integrability and Geometry, vol. 2, paper 068, 17 pages, 2006.

[9] G. H. Maluleke and D. P. Mason, "Derivation of conservation laws for a nonlinear wave equation modelling melt migration using Lie point symmetry generators," Communications in Nonlinear Science and Numerical Simulation, vol. 12, no. 4, pp. 423-433, 2007.

[10] M. Nakayama and D. P. Mason, "Compressive solitary waves in compacting media," International Journal of Non-Linear Mechanics, vol. 26, no. 5, pp. 631-640, 1991.

[11] M. Nakayama and D. P. Mason, "On the existence of compressive solitary waves in compacting media," Journal of Physics A, vol. 27, no. 13, pp. 4589-4599, 1994.

[12] M. Nakayama and D. P. Mason, "On the effect of background voidage on compressive solitary waves in compacting media," Journal of Physics A, vol. 28, no. 24, pp. 7243-7261, 1995. 


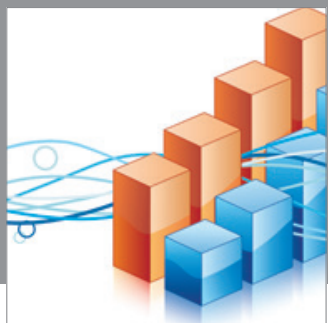

Advances in

Operations Research

mansans

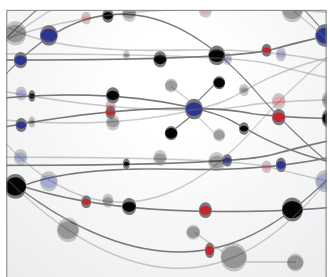

The Scientific World Journal
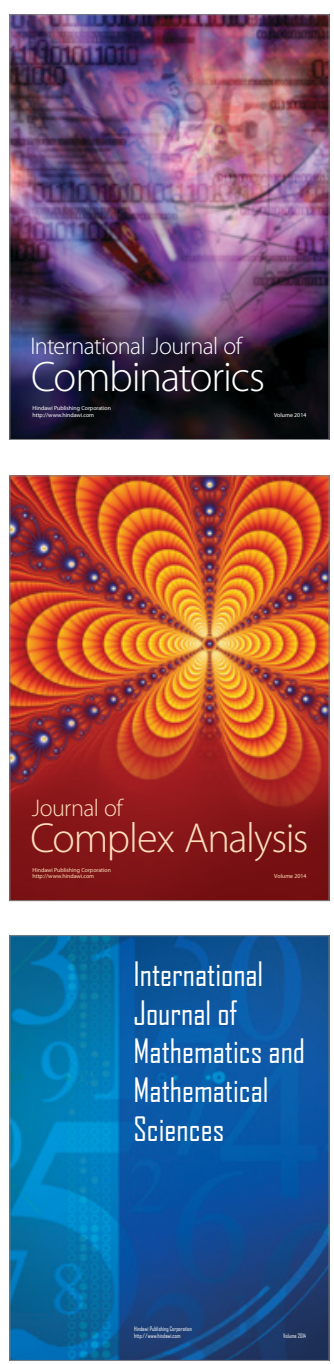
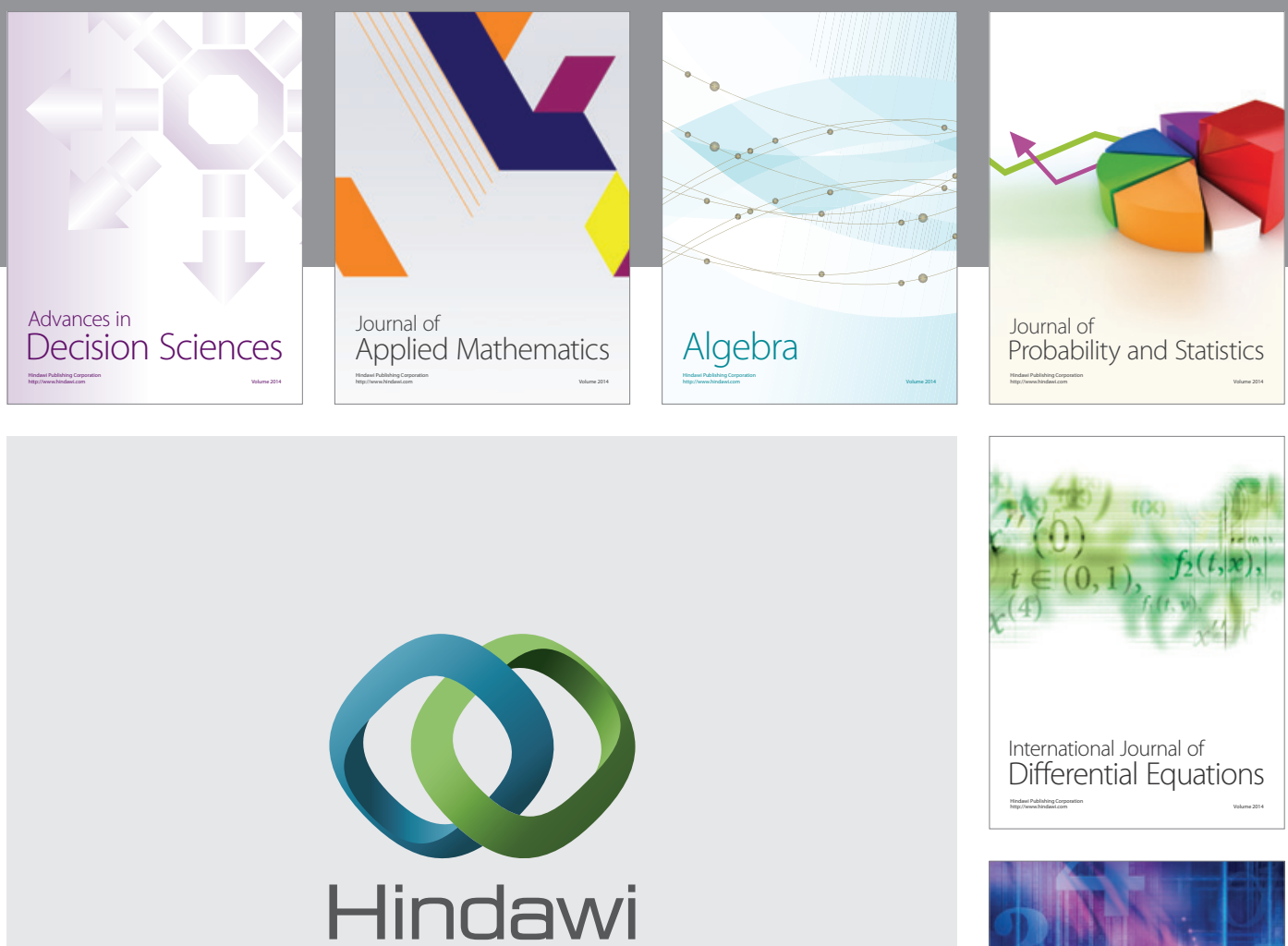

Submit your manuscripts at http://www.hindawi.com
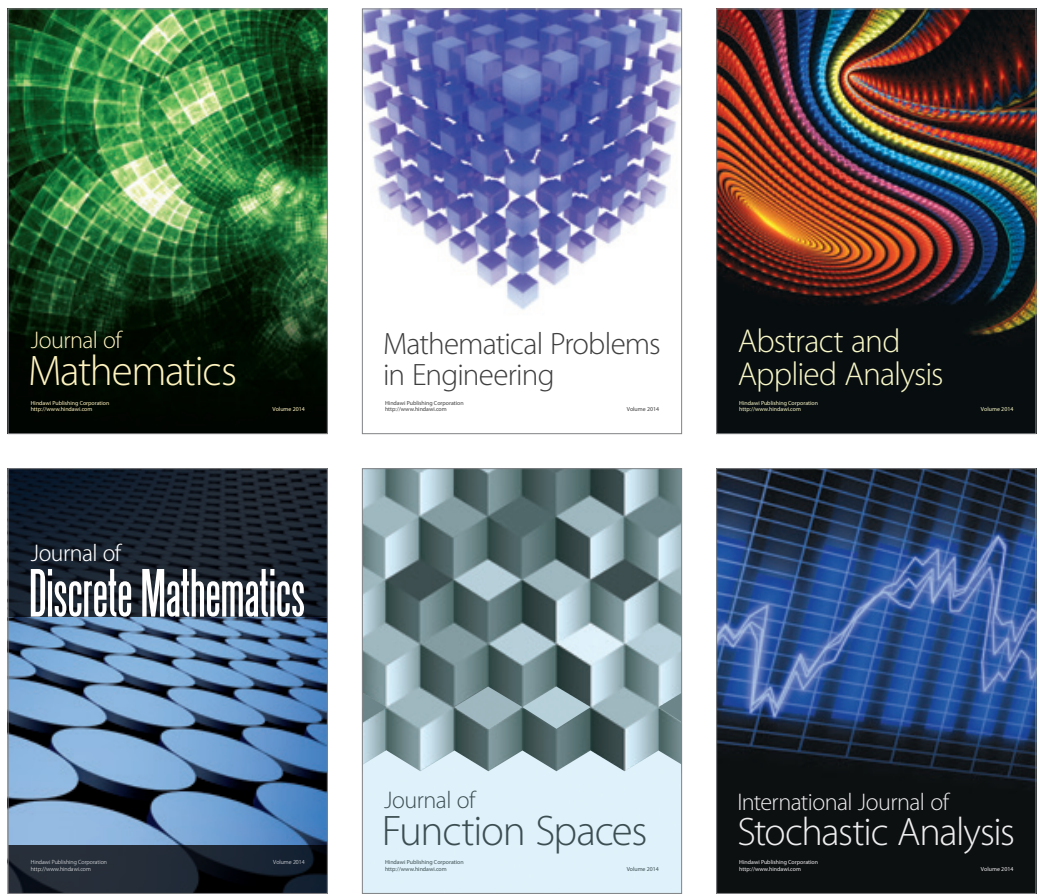

Journal of

Function Spaces

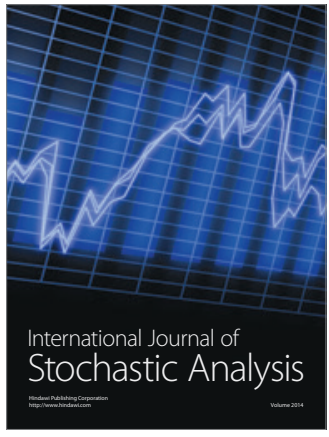

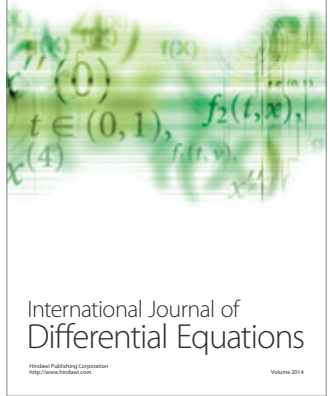
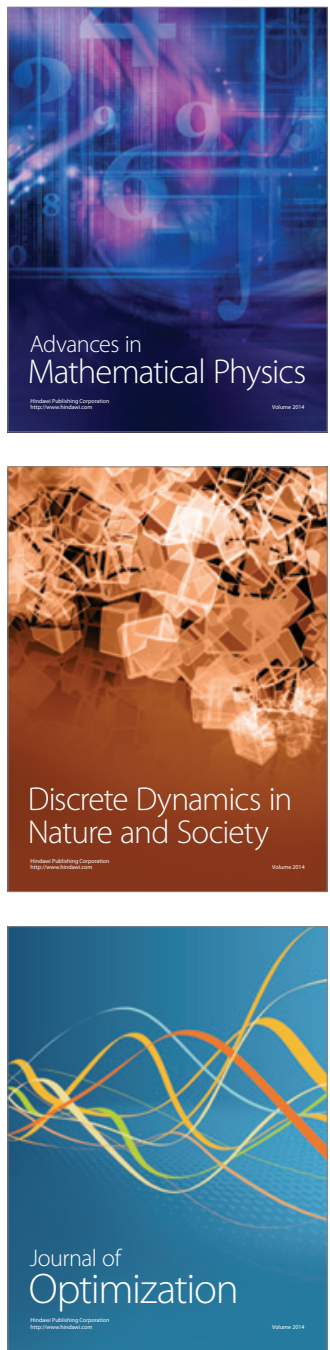